\title{
artigo
}

Gonçalvens, K.K.N.; Araújo, H.V.S.; Morais, C.A.C.; Figueirêdo, T.R.; Moraes, J.C.O.; Bezerra, S.M.M.S.;

Qualidade de vida de profissionais de uma empresa prestadora de serviços gerais e administrativos

\section{Qualidade de vida de profissionais de uma empresa prestadora de serviços gerais e administrativos}

\author{
Quality of life of professionals in a company providing general and administrative services
}

Calidad de vida de los profesionales en una empresa que presta servicios generales y administrativos

\section{RESUMO}

Objetivo: Avaliar a qualidade de vida de funcionários de uma empresa prestadora de serviços gerais e administrativos. Método: Foi realizado um estudo descritivo, transversal com abordagem quantitativa, no qual 66 profissionais terceirizados foram entrevistados utilizando-se um questionário sócio demográfico próprio, o instrumento de qualidade de vida relacionada à saúde SF-36 e avaliação antropométrica. Resultados: Em relação à qualidade de vida relacionada à saúde, os resultados encontrados apresentaram valores médios elevados (acima de 70) para a maioria dos domínios analisados do SF-36. Os resultados indicam que os maiores escores foram obtidos nas dimensões Aspectos Sociais, Aspectos Físicos e Emocionais e os menores escores foram observados nas dimensões Vitalidade e Dor. Conclusão: Reforça-se a importância do enfermeiro reconhecer profissionais em risco ao adoecimento no ambiente de trabalho e intervir através de estratégias de educação em saúde e acompanhamento com vistas à garantia da qualidade de vida do trabalhador.

DESCRITORES: Qualidade de Vida; Saúde do Trabalhador; Fatores de Risco; Doenças Cardiovasculares; Cuidados de Enfermagem.

\section{ABSTRACT}

Objective: To assess the quality of life of employees of a company providing general and administrative services. Method: A descriptive, cross-sectional study with a quantitative approach was carried out, in which 66 outsourced professionals were interviewed using their own socio-demographic questionnaire, the SF-36 health-related quality of life instrument and anthropometric assessment. Results: Regarding health-related quality of life, the results found showed high average values (above 70) for most of the SF-36 domains analyzed. The results indicate that the highest scores were obtained in the dimensions Social Aspects, Physical and Emotional Aspects and the lowest scores were observed in the dimensions Vitality and Pain. Conclusion: It reinforces the importance of nurses to recognize professionals at risk of illness in the work environment and intervene through health education and monitoring strategies with a view to guaranteeing the worker's quality of life.

DESCRIPTORS: Quality of life; Occupational Health; Risk Factors; Cardiovascular Diseases; Nursing Care.

\section{RESUMEN}

Objetivo: Evaluar la calidad de vida de los empleados de una empresa que brinda servicios generales y administrativos. Método: Se realizó un estudio descriptivo, transversal con enfoque cuantitativo, en el que se entrevistaron a 66 profesionales subcontratados mediante cuestionario sociodemográfico propio, instrumento de calidad de vida relacionada con la salud SF-36 y evaluación antropométrica. Resultados: En cuanto a la calidad de vida relacionada con la salud, los resultados encontrados mostraron valores promedio altos (superiores a 70) para la mayoría de los dominios del SF-36 analizados. Los resultados indican que las puntuaciones más altas se obtuvieron en las dimensiones Aspectos sociales, Aspectos físicos y emocionales y las puntuaciones más bajas se observaron en las dimensiones Vitalidad y Dolor. Conclusión: Refuerza la importancia de las enfermeras para reconocer a los profesionales en riesgo de enfermedad en el ambiente laboral e intervenir a través de estrategias de educación y seguimiento en salud con miras a garantizar la calidad de vida del trabajador.

DESCRIPTORES: Calidade de vida; Salud Laboral; Factores de Riesgo; Enfermedades Cardiovasculares; Atención en enfermería.

RECEBIDO EM: 27/01/2021 APROVADO EM: 17/02/2021

\section{Karyne Kirley Negromonte Gonçalves}

Enfermeira. Mestre em Enfermagem pelo Programa Associado de Pós Graduação em Enfermagem UPE/UEPB.

ORCID: 0000-0002-0205-4112 
Hirla Vanessa Soares de Araújo

Enfermeira. Doutoranda em Enfermagem pelo Programa Associado de Pós Graduação em Enfermagem UPE/UEPB. ORCID: 0000-0002-9995-7288

\section{Camila Abrantes Cordeiro Morais}

Enfermeira. Doutoranda em Enfermagem pelo Programa Associado de Pós Graduação em Enfermagem UPE/UEPB. ORCID: 000-0003-3780-9340

\section{Thaisa Remigio Figueirêdo}

Enfermeira. Doutora pelo Programa Associado de Pós Graduação em Enfermagem UPE/UEPB.

ORCID: 0000-0002-0513-5815

\section{Janaíne Chiara Oliveira Moraes}

Enfermeira. Doutora pelo Programa Associado de Pós Graduação em Enfermagem UPE/UEPB.

ORCID: 0000-0002-0266-4762

\section{Simone Maria Muniz da Silva Bezerra}

Enfermeira. Pós-Doutora. Docente do Programa Associado de Pós Graduação em Enfermagem UPE/UEPB.

ORCID: 0000-0002-0974-1409

\section{INTRODUÇÃO}

$\mathbf{N}$ o Brasil, as doenças cardiovasculares lideram o número de mortes, sendo responsáveis por um alto índice de mortalidade, evento justificado pela maior prevalência dos fatores de risco cardiovasculares ${ }^{(1,2)}$. Tais fatores de risco cardiovasculares se expressam em hábitos de vida, como o tabagismo, o sedentarismo, dieta, obesidade e o sobrepeso e as características inatas ou herdadas, como o diabetes mellitus a síndrome metabólica, dislipidemias e a hipertensão arterial sistêmica ${ }^{(3)}$.

No ambiente de trabalho, estes fatores de risco podem afetar diretamente a qualidade de vida do portador, interferindo de forma negativa em suas atividades de vida diárias podendo impactar negativamente sobre a saúde do trabalhador ${ }^{(4)}$.

As questôes socioeconômicas e ocupacionais no ambiente profissional também podem estar relacionadas à qualidade de vida e ao maior risco de desenvolvimento de doenças cardiovasculares, além de serem influenciadas pelo ritmo do trabalho e estrutura e organização do ambiente no qual o profissional está inserido ${ }^{(5)}$.

A Qualidade de Vida (QV), segundo a Organização Mundial de Saúde (OMS), não possui uma definição exata. Por isso, o World Health Organization Quality of Life (WHOQOL) definiu Qualidade de Vida como a "percepção do indivíduo de sua posição na vida no contexto da cultura e sistemas de valores em que ele vive e em relação aos seus objetivos, expectativas, padrões e preocupações" (6).

De acordo com a OMS, a saúde não é o centro da QV. Ela é considerada abstrata, subjetiva e multidimensional por envolver vários aspectos da vida humana, como relações sociais, saúde, família, trabalho, meio ambiente, dentre outros. É um processo dinâmico que pode ser influenciado por aspectos culturais, religiosos, éticos e valores sociais ${ }^{(7)}$.

No ambiente profissional considera-se o conceito de Qualidade de Vida no Trabalho (QVT), que é uma compreensão das condições de vida no ambiente laboral, incluindo aspectos de bem-estar, garantia da saúde, segurança física, mental, social e capacitação para realizar tarefas com segurança e bom uso de energia pessoal ${ }^{(8)}$.

O hospital, por ser um ambiente insalubre, expõe o trabalhador a inúmeros riscos, como físicos, químicos, fisiológicos, psíquicos, mecânicos e biológicos. Desta forma, torna-se um ambiente ocupacional que deixa o profissional vulnerável a aquisição de doenças, dentre elas, as do aparelho cardiovascular ${ }^{(9)}$.

Cada vez são mais claras as consequências que as condições de trabalho e a carga horária exigida acarretam na saúde física do trabalhador. Cerca de $29 \%$ da população ativa trabalha por turnos, onde os efei- tos da intolerância ao trabalho assumem uma elevada importância no âmbito da saúde ocupacional ${ }^{(10)}$.

A sobrecarga quantitativa de trabalho, a duração, falta de autonomia, a presença de riscos físicos, químicos e biológicos, a insuficiência de recursos, a responsabilidade por pessoas, as relações de trabalho e suporte social no local de emprego, a remuneração, a segurança no vínculo de trabalho, as perspectivas de promoção, o quadro familiar, o conflito casa-trabalho, o quadro social e pessoal são considerados fatores de risco para o adoecimento nestes profissionais ${ }^{(10)}$.

Dentro desse contexto, a exposição contínua às cargas de trabalho gera desgaste no trabalhador, podendo evoluir para o adoecimento. Desta forma, é necessário que o trabalhador compreenda as peculiaridades da profissão, e aproprie-se de estratégias que possam reduzir o desgaste profissional, buscando a preservação da sua saúde ${ }^{(11)}$.

Nesse sentido, o presente estudo objetivou avaliar a qualidade de vida de funcionários de uma empresa prestadora de serviços gerais e administrativos.

\section{MÉTODOS}

Trata-se de um estudo transversal, descritivo, com abordagem quantitativa. A pesquisa foi realizada no ambulatório de Cardiologia do Pronto Socorro Cardiológico de Pernambuco Professor Luiz Tavares, 


\section{artigo}

PROCAPE/UPE, Recife/PE, referência em Cardiologia que abrange atendimento ao Recife e regiões Norte e Nordeste do País, no período de Agosto a Novembro de 2017.

A amostra foi composta por 66 profissionais terceirizados da empresa prestadora de serviços especializados ao PROCAPE distribuídos em serviços gerais (encanador, pintor, eletricista, auxiliar de limpeza) e serviços administrativos (secretário, recepcionista). Incluíram-se funcionários de ambos os sexos, com idade igual ou superior a 18 anos e que concordassem em participar mediante a assinatura do Termo de Consentimento Livre e Esclarecido. Foram excluídos os participantes que possuíam comprometimento cognitivo que redundasse em comunicação verbal prejudicada e que faziam uso de antidepressivos.

Os instrumentos de coleta de dados foram compostos por um questionário próprio com dados sócio demográficos, econômicos (gênero, idade, raça, estado civil, renda familiar mensal em salários-mínimos vigentes no ano de 2017- $\mathrm{R} \$ 937,00$ ), escolaridade, procedência e clínicos (hábitos de vida, antecedentes pessoais e familiares, doenças pré-existentes).

Para avaliar a qualidade de vida dos profissionais da empresa terceirizada, foi utilizado o Instrumento de Qualidade de Vida Relacionado à Saúde-SF36 (Anexo A). Trata-se de um questionário multidimensional traduzido e validado no Brasil, que considera a percepção dos indivíduos quanto ao seu próprio estado de saúde. É constituído por
36 itens subdivididos em oito domínios: Capacidade Funcional, Aspectos Físicos, Dor, Estado Geral de Saúde, Vitalidade, Aspectos Sociais, Aspectos Emocionais e Saúde Mental ${ }^{(12)}$. Os dados são analisados a partir da transformação das respostas de cada domínio em escore numa escala que varia de 0 (zero) a 100 (cem), com os escores mais altos indicando melhor estado de saúde e os menores escores correspondendo ao pior estado geral de saúde ${ }^{(13)}$.

As variáveis foram analisadas de forma descritiva e foram processadas e analisadas com o uso do software IBM SPSS na versão 20. 0. Utilizou-se o teste $\mathrm{t}$-Student $\mathrm{e}$ adotou-se $\mathrm{p}<0,05$.

Esta pesquisa seguiu as normas disciplináveis da Resolução № 510 de 07 de Abril de 2016, do Conselho Nacional de Saúde do Ministério da Saúde (14), tendo sida aprovada pelo comitê de ética e pesquisa do complexo Hospital Universitário Oswaldo Cruz/ Pronto Socorro Cardiológico de Pernambuco (CAE: 68383817000005192) e Parecer Consubstanciado do Comitê de Ética e Pesquisa (Número do Parecer: 2.142.908).

\section{RESULTADOS}

Um total de 66 profissionais terceirizados (auxiliar de limpeza, serviços gerais, secretários, atendentes, supervisores) participou do estudo. A amostra foi composta predominantemente por participantes do sexo masculino (57,6\%), com média de idade de

\begin{tabular}{|c|c|c|c|c|}
\hline DOMíNIO & MÉDIA & DESVIO-PADRÃO & MÍNIMO & MÁXIMO \\
\hline Estado Geral de Saúde & 77,83 & $\pm 17,5$ & 30 & 100 \\
\hline Capacidade Funcional & 88,11 & $\pm 16,30$ & 30 & 100 \\
\hline Aspectos Físicos & 89,85 & $\pm 21,95$ & 10 & 100 \\
\hline Aspectos Emocionais & 88,67 & $\pm 22,65$ & 10 & 100 \\
\hline Aspectos Sociais & 93,56 & $\pm 15,69$ & 25 & 100 \\
\hline Dor & 71,03 & $\pm 21,73$ & 32 & 100 \\
\hline Vitalidade & 58,73 & $\pm 15,52$ & 6 & 100 \\
\hline Saúde Mental & 79,55 & $\pm 17,29$ & 32 & 100 \\
\hline
\end{tabular}

38 anos $( \pm 1,189)$, de raça parda $(47,0 \%)$, procedentes da Zona Urbana (97,0\%), com companheiro fixo (54,5\%). A maioria possuía mais de 9 anos de estudo (84,8\%), com renda familiar mensal maior que 2 salários mínimos (78,8\%).

Em relação ao tempo de serviço prestado à instituição, 87,9\% dos participantes possuíam mais de 1 ano de trabalho, 71,2\% desempenham a função de serviços gerais e $66,7 \%$ apresentam em regime de trabalho plantonista.

Em relação aos aspectos clínicos, os fatores de risco para as doenças cardiovasculares mais frequentes foram o uso de bebida alcóolica socialmente $(54,5 \%)$, sedentarismo $(59,1 \%)$ e hipertensão arterial (16,7\%).

Em relação à avaliação da qualidade de vida relacionada à saúde, na tabela 2 está apresentada a pontuação dos oito domínios do Questionário de Qualidade de Vida SF36 expressos em média e desvio padrão e seus respectivos intervalos de confiança. A questão 2 não pertence a nenhum domínio, todavia, analisa a percepção do indivíduo em relação à sua saúde comparada há um ano. Parte dos participantes do estudo (36,4\%) respondeu que está muito melhor ou um pouco melhor. Os demais se dividiram entre os que consideraram sua saúde um pouco pior $(12,1 \%)$ e os que avaliaram que está aproximadamente igual quando comparada há um ano (51,5\%).

Para cada uma das oito dimensões do SF-36 foram calculados os valores da média e desvio padrão. Os valores para todas as dimensões poderiam variar entre 0 e 100, sendo que quanto maior o escore, melhor a condição relacionada àquela dimensão da qualidade de vida. Foram observados escores médios, em geral, acima de 70 para a maioria dos domínios. Considerando-se que o escore em cada domínio pode variar de zero a 100 , os resultados demonstram valores médios elevados na grande maioria dos domínios analisados.

Os resultados indicam que os maiores escores foram obtidos nas dimensões Aspectos Sociais (93,56\%), Aspectos Físicos (89,85\%) e Aspectos Emocionais (88,67\%). Já em relação aos menores escores foram observados nas dimensões Vitalidade (58,73\%) e Dor (71,03\%).

A Tabela 2 mostra os resultados da ava- 
liação da Qualidade de vida pelo SF-36 em função de algumas variáveis sócio demográficas e clínicas. Os dados com significância estatística apresentam $\mathrm{p}<0,05$.

Em relação às variáveis sócio demográficas, os profissionais da ADLIM sem companheiro apresentaram pior avaliação no domínio Aspectos Emocionais ( $\mathrm{p}=0,008$ ), assim como no domínio Aspectos Sociais $(p=0,012)$. Em relação ao tempo de trabalho, profissionais com mais de 1 ano de serviço apresentaram pior avaliação no domínio Estado Geral de Saúde ( $\mathrm{p}=0,021)$. Já em relação às variáveis clínicas, o não uso de bebida alcoólica $(\mathrm{p}=0,001)$ apresentou pior avaliação no domínio Dor.

Em relação ao domínio saúde mental, os profissionais tabagistas $(\mathrm{p}=0,027)$ e diabéti$\cos (\mathrm{p}=0,005)$ apresentaram pior avaliação no domínio saúde mental. Ainda se tratando dos diabéticos, também apresentaram pior avaliação nos domínios Estado geral de saúde $(\mathrm{p}=0,005)$, aspectos funcionais $(\mathrm{p}=0,002) \mathrm{e}$ aspectos emocionais $(\mathrm{p}=<0,001)$.

Em relação ao domínio Capacidade Funcional, os profissionais com índice de massa corporal (IMC) alterado, apresentaram pior avaliação $(\mathrm{p}=0,018)$.

\section{DISCUSSÃO}

Em relação à avaliação da qualidade de vida pelo SF-36, no domínio relacionado aos

\section{Tabela 2 - Avaliação da Qualidade de Vida Relacionada à Saúde pelo SF-36 em função das variáveis sócio demográficas e clínicas (n=66). Recife, PE, Brasil. 2018.}

QUALIDADE DE VIDA RELACIONADA À SAÚDE (MD \pm DP)

\begin{tabular}{|c|c|c|c|c|c|c|c|c|c|c|c|c|c|c|c|c|}
\hline $\begin{array}{c}\text { SÓCIO } \\
\text { DEMO- } \\
\text { GRÁFICA }\end{array}$ & EGS & $\mathrm{P}^{*}$ & CF & $\mathrm{P}^{*}$ & AF & $\mathrm{P}^{*}$ & $\mathrm{AE}$ & $P^{*}$ & DOR & $P^{*}$ & AS & $P^{*}$ & V & $\mathrm{P}^{*}$ & SM & $\mathrm{P}^{*}$ \\
\hline \multicolumn{17}{|c|}{ Com companheiro } \\
\hline $\begin{array}{l}\text { Com com- } \\
\text { panheiro }\end{array}$ & $\begin{array}{c}79,7 \pm \\
17,4\end{array}$ & \multirow{2}{*}{, 342} & $\begin{array}{c}89,3 \pm \\
15,9\end{array}$ & \multirow{2}{*}{, 517} & $\begin{array}{c}94,4 \pm \\
13,5\end{array}$ & \multirow{2}{*}{,055 } & $\begin{array}{c}95,3 \pm \\
11,7\end{array}$ & \multirow{2}{*}{, 008} & $\begin{array}{c}75,5 \pm \\
22,9\end{array}$ & \multirow{2}{*}{, 148} & $\begin{array}{c}97,29 \pm \\
7,6\end{array}$ & \multirow{2}{*}{012} & $\begin{array}{c}59,6 \pm \\
17,3\end{array}$ & \multirow{2}{*}{, 616} & $\begin{array}{c}80,9 \pm \\
14,0\end{array}$ & \multirow{2}{*}{, 476} \\
\hline $\begin{array}{l}\text { Sem Com- } \\
\text { panheiro }\end{array}$ & $\begin{array}{c}75,5 \pm \\
17,7\end{array}$ & & $\begin{array}{c}86,6 \pm \\
16,8\end{array}$ & & $\begin{array}{c}84,0 \pm \\
28,4\end{array}$ & & $\begin{array}{c}80,6 \pm \\
29,3\end{array}$ & & $\begin{array}{c}66,8 \pm \\
19,7\end{array}$ & & $\begin{array}{c}88,3 \pm \\
20,7\end{array}$ & & $\begin{array}{c}57,6 \pm \\
13,1\end{array}$ & & $\begin{array}{c}77,8 \pm \\
20,6\end{array}$ & \\
\hline \multicolumn{17}{|c|}{ Tempo de Trabalho } \\
\hline Até 1 ano & $\begin{array}{c}91,1 \pm \\
5,4\end{array}$ & \multirow{2}{*}{,021 } & $\begin{array}{c}87,5 \pm \\
20,3\end{array}$ & \multirow{2}{*}{912} & $\begin{array}{c}90,6 \pm \\
26,5\end{array}$ & \multirow{2}{*}{,900 } & $\begin{array}{c}87,4 \pm \\
24,8\end{array}$ & & $\begin{array}{c}84,2 \pm \\
21,9\end{array}$ & \multirow{2}{*}{,876 } & $\begin{array}{c}90,6 \pm \\
26,5\end{array}$ & \multirow{2}{*}{, 577} & $\begin{array}{c}66,2 \pm \\
11,2\end{array}$ & \multirow{2}{*}{, 145} & $\begin{array}{c}87,5 \pm \\
16,0\end{array}$ & \multirow{2}{*}{, 167} \\
\hline $\begin{array}{c}\text { Mais de } 1 \\
\text { ano }\end{array}$ & $\begin{array}{c}76,0 \pm \\
17,8\end{array}$ & & $\begin{array}{l}88,1 \pm \\
15,88\end{array}$ & & $\begin{array}{c}89,5 \pm \\
21,6\end{array}$ & & $\begin{array}{c}88,8 \pm \\
22,5\end{array}$ & & $\begin{array}{c}69,2 \pm \\
21,2\end{array}$ & & $\begin{array}{c}93,9 \pm \\
13,9\end{array}$ & & $\begin{array}{c}57,6 \pm \\
15,8\end{array}$ & & $\begin{array}{c}78,4 \pm \\
17,3\end{array}$ & \\
\hline \multicolumn{17}{|c|}{ Qualidade de Vida Relacionada à Saúde (md \pm dp) } \\
\hline $\begin{array}{l}\text { Variável } \\
\text { clínica }\end{array}$ & EGS & $\mathrm{p}^{*}$ & CF & $\mathrm{p}^{*}$ & AF & $\mathrm{p}^{*}$ & $\mathrm{AE}$ & $\mathrm{p}^{*}$ & Dor & $\mathrm{p}^{*}$ & AS & $\mathrm{p}^{*}$ & V & $\mathrm{p}^{*}$ & SM & $\mathrm{p}^{*}$ \\
\hline $\begin{array}{l}\text { Uso de } \\
\text { bebida }\end{array}$ & $\begin{array}{c}77,7 \pm \\
18,7\end{array}$ & \multirow{3}{*}{,978 } & $\begin{array}{c}89,3 \pm \\
17,2\end{array}$ & \multirow{3}{*}{,581 } & $\begin{array}{c}95,3 \pm \\
17,3\end{array}$ & \multirow{3}{*}{,058 } & $\begin{array}{c}94,4 \pm \\
15,3\end{array}$ & & $\begin{array}{c}80,7 \pm \\
21,4\end{array}$ & \multirow{3}{*}{,001 } & $\begin{array}{c}95,8 \pm \\
10,5\end{array}$ & \multirow{3}{*}{,286 } & $\begin{array}{c}61,8 \pm \\
14,5\end{array}$ & & $\begin{array}{c}81,3 \pm \\
16,1\end{array}$ & \multirow{3}{*}{,448 } \\
\hline Não usa & & & & & & & & \multirow[t]{2}{*}{,059 } & & & & & & ,139 & & \\
\hline $\begin{array}{c}\text { bebida } \\
\text { alcoólica }\end{array}$ & $\begin{array}{c}77,8 \pm \\
16,7\end{array}$ & & $\begin{array}{c}87,0 \pm \\
15,6\end{array}$ & & $\begin{array}{c}85,0 \pm \\
24,6\end{array}$ & & $\begin{array}{c}83,8 \pm \\
26,5\end{array}$ & & $\begin{array}{c}62,9 \pm \\
18,7\end{array}$ & & $\begin{array}{c}91,6 \pm \\
18,8\end{array}$ & & $\begin{array}{c}56,1 \pm \\
15,9\end{array}$ & & $\begin{array}{c}78,0 \pm \\
18,3\end{array}$ & \\
\hline $\begin{array}{l}\text { Não Taba- } \\
\text { gistas }\end{array}$ & $\begin{array}{c}78,8 \pm \\
16,8\end{array}$ & \multirow{2}{*}{,281 } & $\begin{array}{c}87,8 \pm \\
16,1\end{array}$ & \multirow{2}{*}{,729 } & $\begin{array}{c}91,2 \pm \\
19,8\end{array}$ & \multirow{2}{*}{ 115 } & $\begin{array}{c}90,3 \pm \\
19,9\end{array}$ & & $\begin{array}{c}71,5 \pm \\
21,9\end{array}$ & \multirow{2}{*}{,098 } & $\begin{array}{c}93,8 \pm \\
13,9\end{array}$ & & $\begin{array}{c}60,0 \pm \\
14,3\end{array}$ & & $\begin{array}{c}81,2 \pm \\
16,7\end{array}$ & \multirow{2}{*}{,027 } \\
\hline Tabagistas & $\begin{array}{c}70,6 \pm \\
22,1\end{array}$ & & $\begin{array}{c}90,0 \pm \\
18,7\end{array}$ & & $\begin{array}{c}78,1 \pm \\
33,9\end{array}$ & & $\begin{array}{c}76,2 \pm \\
36,1\end{array}$ & & $\begin{array}{c}67,3 \pm \\
21,3\end{array}$ & & $\begin{array}{c}90,6 \pm \\
26,5\end{array}$ &, 577 & $\begin{array}{c}49,5 \pm \\
21,3\end{array}$ & ,073 & $\begin{array}{c}67,0 \pm \\
16,8\end{array}$ & \\
\hline $\begin{array}{c}\text { Sem } \\
\text { Diabetes }\end{array}$ & $\begin{array}{c}78,5 \pm \\
16,6\end{array}$ & \multirow{2}{*}{,005 } & $\begin{array}{c}87,9 \pm \\
16,6\end{array}$ & \multirow{2}{*}{,467 } & $\begin{array}{c}90,6 \pm \\
20,7\end{array}$ & & $\begin{array}{c}89,8 \pm \\
20,5\end{array}$ & & $\begin{array}{c}71,3 \pm \\
21,7\end{array}$ & & $\begin{array}{c}93,4 \pm \\
15,7\end{array}$ & & $\begin{array}{c}59,0 \pm \\
15,4\end{array}$ & & $\begin{array}{c}80,2 \pm \\
16,6\end{array}$ & \\
\hline $\begin{array}{c}\text { Com } \\
\text { Diabetes }\end{array}$ & $30,0^{* *}$ & & $100,0^{* *}$ & & $25,0^{* *}$ & ,002 & $10,0^{* *}$ & $<, 001$ & $52,0^{*}$ & 382 & $100,0^{* *}$ & 683 & $40,0^{* *}$ & 227 & $30,0^{* *}$ & ,005 \\
\hline $\begin{array}{l}\text { IMC Nor- } \\
\text { mal }\end{array}$ & $\begin{array}{c}84,1 \pm \\
18,4\end{array}$ & & $\begin{array}{c}95,5 \pm \\
8,3\end{array}$ & & $\begin{array}{c}90,7 \pm 1 \\
9,0\end{array}$ & & $\begin{array}{c}86,4 \pm \\
23,8\end{array}$ & & $\begin{array}{c}74,7 \pm \\
20,0\end{array}$ & & $\begin{array}{c}94,7 \pm \\
12,0\end{array}$ & & $\begin{array}{c}60,5 \pm \\
19,4\end{array}$ & & $\begin{array}{c}82,3 \pm \\
17,5\end{array}$ & \\
\hline $\begin{array}{l}\text { IMC alte- } \\
\text { rado }\end{array}$ & $\begin{array}{c}75,2 \pm \\
16,7\end{array}$ &, 062 & $\begin{array}{c}85,1 \pm \\
17,8\end{array}$ & (018 & $\begin{array}{c}89,2 \pm \\
23,3\end{array}$ & , 801 & $\begin{array}{c}89,5 \pm \\
22,3\end{array}$ &, $6<0$ & $\begin{array}{c}69,5 \pm \\
22,4\end{array}$ & , $3 / 6$ & $\begin{array}{c}93,0 \pm \\
17,0\end{array}$ &, 102 & $\begin{array}{c}56,9 \pm \\
13,8\end{array}$ &, 542 & $\begin{array}{c}78,4 \pm \\
17,2\end{array}$ &, 412 \\
\hline
\end{tabular}


aspectos sociais, que refletem a capacidade de relacionamento dos participantes, o resultado desta avaliação revelou alto escore neste domínio. Estudo que avaliou a qualidade de vida e sintomas osteomusculares em trabalhadores de higiene e limpeza hospitalar vai de encontro aos resultados desta pesquisa, pois apresentaram pior pontuação neste domínio ${ }^{(15)}$.

Em relação escores foram observados nas dimensões Vitalidade e Dor valores inferiores. $\mathrm{O}$ resultado vai de encontro aos encontrados no estudo de Registro Brasileiro de Marcapassos, Ressincronizadores e Desfibriladores ${ }^{(16)}$ que evidenciou que o domínio ficou com valores acima da média. Sabe-se que o mesmo pode influenciar na capacidade funcional do indivíduo, pois pode apresentar impacto com prejuízo negativo no desempenho das atividades diárias e/ou profissionais. Observa-se que quanto mais o indivíduo sente dor, possivelmente, mais comprometida encontra-se a sua qualidade de vida, o que pode acarretar em impacto negativo nas atividades diárias e/ou profissionais ${ }^{(16,17)}$.

Em relação à avaliação da qualidade de vida em função das variáveis sociodemográficas dos participantes do estudo, no tocante ao estado civil, os profissionais avaliados sem companheiros, apresentaram pior avaliação nos domínios aspectos emocionais ( $\mathrm{p}=0,008$ ) e sociais $(\mathrm{p}=0,012)$. O resultado diverge do encontrado em estudo sobre a influência da hipertensão arterial na qualidade de vida (18), o qual evidenciou que os participantes que possuíam cônjuge apresentaram melhores médias de qualidade de vida relacionado à saúde nos domínios capacidade funcional, capacidade física, aspectos físicos e aspectos emocionais $(\mathrm{p}<0,05)$ quando foram comparados aos sujeitos que não os tinham.

Em relação ao tempo de trabalho, os participantes que prestam serviços há mais de 1 ano na empresa, apresentaram pior avaliação no domínio Estado Geral de Saúde $(\mathrm{p}=0,021)$. O trabalho é uma atividade que pode ocupar grande parcela do tempo de cada indivíduo e do seu convívio em sociedade e nem sempre possibilita realização profissional, pode ao contrário, causar problemas desde insatisfação até exaustão, interferindo no estado geral de saúde do trabalhador ${ }^{(18)}$.
Em relação à avaliação QV em função das variáveis clínicas dos participantes do estudo, no tocante ao uso de bebida alcoólica de forma social pelos profissionais, o não uso da mesma evidenciou pior avaliação no domínio Dor $(\mathrm{p}=0,001)$. No entanto, achados diferentes foram evidenciados em indivíduos que apresentaram um consumo excessivo de álcool em relação aos que não consomem ou consomem moderadamente, obtendo pior qualidade de vida na dimensão capacidade funcional ${ }^{(19)}$.

Em relação ao domínio Saúde Mental, os profissionais tabagistas apresentaram pior avaliação $(p=0,027)$ corroborando com os achados encontrados no estudo que investigou o impacto dos fatores de risco para doenças crônicas não transmissíveis na $\mathrm{QV}$ dos indivíduos, no qual o tabagismo apresentou-se como o fator de risco de maior impacto na QV com piores escores nos domínios aspectos físicos, estado geral de saúde, aspectos sociais e saúde mental ${ }^{(20)}$.

\section{Em relação ao}

domínio Capacidade

Funcional, os

participantes

que possuíam

\section{IMC alterado,}

\section{apresentaram pior \\ avaliação $(p=0,018)$}

Em contrapartida, estudo evidenciou que ao avaliar a qualidade de vida em relação à gravidade da dependência de tabaco de não-fumantes, ex-fumantes, tabagistas leves (consumo inferior a 15 cigarros por dia), moderados (consumo de 15-24 cigarros por dia) e graves (consumo igual ou superior a 25 cigarros/dia) foi observado prejuízo dos tabagistas moderados e graves em todas as dimensões do SF- 36, quando comparados aos não-fumantes ${ }^{(21)}$.
Em relação aos profissionais diabéticos, os mesmos apresentaram piores escores nos domínios Estado geral de Saúde ( $\mathrm{p}=0,005)$, aspectos funcionais $(\mathrm{p}=0,002)$, aspectos emocionais $(p<0,001)$ e saúde mental $(p=0,005)$. Semelhante a estes achados, o estudo que avaliou a qualidade de vida de 40 pacientes diabéticos tipo 2 e a prevalência de déficit sensitivo em membros inferiores revela que os diabéticos tendem a ter alterações na saúde física ou algum problema emocional que interferem negativamente no seu trabalho ou em outra atividade regular diária como depressão ou complicações decorrentes da diabetes ${ }^{(22)}$.

Em relação ao domínio Capacidade Funcional, os participantes que possuíam IMC alterado, apresentaram pior avaliação $(p=0,018)$. Achados semelhantes foram encontrados no estudo que avaliou a qualidade de vida e nível de atividade física de profissionais de saúde de unidades de terapia intensiva, evidenciando que os indivíduos considerados ativos apresentaram maiores pontuações nos domínios capacidade funcional quando comparados aos inativos com IMC elevados ${ }^{(23)}$.

No que se refere à $\mathrm{QV}$ relacionada à saúde avaliada pelo SF-36, o resultado pode ser justificado pelo fato de os participantes, apesar de apresentarem fatores de risco para o desenvolvimento da doença cardiovascular, ainda não têm a doença instalada, e, portanto, é esperado que a boa qualidade de vida seja mantida no intervalo de um ano para o outro.

\section{CONCLUSÃO}

Os trabalhadores da empresa prestadora de serviços especializados apresentam comorbidades, além de fatores de risco cardiovasculares, que se não forem intervindos precocemente podem levar às complicações cardiovasculares. Neste contexto, a prevenção é evidenciada como a melhor terapêutica para o não surgimento de desfechos cardíacos.

O instrumento de Qualidade de Vida Relacionado à Saúde SF-36 foi de avaliar com eficácia a qualidade de vida em profissionais terceirizados. As questôes que en- 
volvem a qualidade de vida possibilitaram a reflexão sobre a real necessidade do profissional terceirizado em relação à sua con- dição de saúde, o que pode, diretamente, auxiliar os profissionais de saúde envolvidos no planejamento de reabilitação, for- necendo, assim, subsídios para a implantação de uma assistência voltada para as reais necessidades desses trabalhadores.

\section{REFERÊNCIAS}

1. Sociedade Brasileira de Cardiologia. Doenças cardiovasculares mata mais de 30 mil pessoas só em Janeiro de 2017. Federação Médica Brasileira. Brasil. [Internet] 2017. [Acesso em 25 jan 2020]. Disponivel em: http://portalfmb.org.br/2017/02/08/doencas-cardiovasculares-mata-mais-de-30-mil-pessoas-so-em-janeirode-2017/.

2. Guimarães RM, Andrade SSCA, Machado EL, Bahia CA, Oliveira MM, Jacques FVL. Diferenças regionais na transição da mortalidade por doenças cardiovasculares no Brasil, 1980 a 2012. Rev Panam Salud Publica. 2015; 37(2): 83-9.

3. Arend MC, Pereira JO, Markoski, MM. O Sistema CRISPR/Cas9 e a Possibilidade de Edição Genômica para a Cardiologia. Arq. Bras. Cardiologia. 2017; 108(1): 81-3. DOI: DOI: 10.5935/abc.20160200.

4. Sadir MA, Bignotto MM, Lipp MEN. Stress e qualidade de vida: influência de algumas variáveis pessoais: influência de algumas variáveis pessoais. Paidéia. 2010; 20(45): 73-81. DOl: http://dx.doi. org/10.1590/s0103-863×2010000100010.

5. Martins CCF, Santos VEP, Pereira MS, Santos NP. Relacionamento interpessoal da equipe de enfermagem $x$ estresse: limitações para a prática. Cogitare Enferm. 2014; 19(2): 309-15. DOI: http://dx.doi. org/10.5380/ce.v19i2.36985.

6. Francisco MS, Azevedo DSB, Campos MTS, Rodrigues RP. Determinantes e condicionantes da vulnerabilidade da qualidade de vida da equipe de enfermagem na assistência em saúde mental e psiquiátrica. Rev. Cientifica Persp Online: bio\& saúde. 2015; 18(5): 45-46. DOI: https://doi.org/10.25242/88685182015773.

7. Gouveia AMA, Fagundes CR, Macêdo SS, Junior AJC, Casa NLL, Cruz RS. Análise da Qualidade de Vida no trabalho em caminhoneiros de uma indústria petrolífera. Rev Eletr Saúde e Ciên. 2012; 2(2): 30-51.

8. Hipolito MCV, Masson VA, Monteiro MI, Gutierrez GL. Quality of working life: assessment of intervention studies. Ver Bras Enferm. 2017; 70(1): 178-86. DOI: http://dx.doi.org/ 10.1590/0034-71672015-0069.

9. Silva CDL, Pinto WM. Riscos ocupacionais no ambiente hospitalar: fatores que favorecem a sua ocorrência na equipe de enfermagem. Saúde Coletiva em Debate. 2012; 2(1): 95-105.

10. Oliveira V, Pereira T. Ansiedade, depressão e burnout em enfermeiros - Impacto do trabalho por turnos. Revista de Enfermagem Referência. 2012; SériellI(7); 43-54. DOI: http://dx.doi. org/10.12707/RIII1175

11. Carvalho DP, Rocha LP, Barlem JGT, Dias JS, Schallenberger CD. Cargas de trabalho e a saúde do trabalhador de enfermagem: revisão integrativa: influência de algumas variáveis pessoais. Cogitare Enferm. 2017; 22(1): 01-11. DOI: http://dx.doi.org/10.5380/ ce.v22i1.46569.

12. Cabral DL. Comparação dos Instrumentos SF-36 e perfil de saúde de Nottingham para avaliação da Qualidade de Vida de indivíduos pós Acidente Vascular Encefálico. Dissertação (Mestrado em Fisioterapia). Universidade Federal de Pernambuco, PernambuCo, 616.81 CDD (22 ed). CCS2011-032. 2011.

13. Laguardia J, Campos MR, Travassos C, Najar AL, Anjos LA, Vasconcellos MM. Dados normativos brasileiros do questionário Short Form-36 versão 2. Rev bras epidemiol. 2013; 16(4): 889-97 889. DOl: https://doi.org/10.1590/S1415-790X2013000400009.

14. Ministério da Saúde (BR). Conselho Nacional de Saúde. Resolução $n^{\circ} 466$, de 12 de dezembro de 2012. Aprova normas regulamentadoras de pesquisas envolvendo seres humanos. Brasilia; 2012.

15. Martarello NA, Benatti MCC. Qualidade de vida e sintomas osteomusculares em trabalhadores de higiene e limpeza hospitalar. Ver Esc Enferm USP. 2009; 43(2). DOI: http://dx.doi.org/10.1590/ 50080-62342009000200023.

16. Pachón-mateos JC, Pereira WL, Junior-Batista WD, Pachón-mateos JC, Pachón-mateo EI, Vargas RNA, et al. RBM-Registro Brasileiro de Marcapassos, Ressincronizadores e Desfibriladores. Relampa. 2013; 26(1): 39-49.

17. Gomes TB, Gomes LS, Antônio IHF, Barroso TL, Cavalcante AMRZ, Stival MM, et al. Avaliação da qualidade de vida pós implante de marca-passo cardíaco artificial. Rev. Eletr. Enf. 2011; 13(4): 735-42.

18. Carvalho MVC, Siqueira LB, Sousa ALL, Jardim PCBVJ. A Influência da Hipertensão Arterial na Qualidade de Vida. Universidade Federal de Goiás, Goiania, GO. Liga de Hipertensão - Brasil. Arq. Bras. Cardiol. 2013; 100(2). DOI: http://dx.doi.org/10.5935/ abc.20130030

19. Trigo TR, Teng CT, Hallak JEC. Síndrome de burnout ou estafa profissional e os transtornos psiquiátricos. Rev Psiq Clín. 2007;34(5):223-33. DOl: https://doi.org/10.1590/S010160832007000500004 .

20. Campos MO, Neto JFR, Silveira MF, Neves DMR, Vilhena JM, Oliveira JF, Magalhães JC, Drumond D. Impacto dos fatores de risco para doenças crônicas não transmissíveis na qualidade de vida. Ciência \& Saúde Coletiva. 2013; 18(3): 873-882. DOI: http://dx.doi. org/10.1590/S1413-81232013000300033.

21. Lima MBP, Ramos D, Freire APCF, Uzeloto JS, Silva BLM, Ramos EMC. Qualidade de vida de tabagistas e sua correlação com a carga tabagística. Fisioter Pesqui. 2017; 24(3): 273-9. DOl: http://dx.doi. org/10.1590/1809-2950/16711324032017.

22. Silva HGN, Lopes RMAL, Feitosa MCP, Souza KF, Oliveira RA. Avaliação da qualidade de vida de pacientes diabéticos tipo 2 e a prevalência de déficit sensitivo em membros inferiores. R. bras. Qual. Vida. 2017; 9(2): 165-77. DOl: 10.3895/rbqv.v9n2.6033.

23. Neto ACFA, Araújo RC, Pitangui ACR, Menezes LC, França EETF, Costa ECC, Andrade FMD, Junior MAVC. Qualidade de vida e nível de atividade física de profissionais de saúde de unidades de terapia intensiva. Ver Bras Ativ Fis Saúde. 2013; 18(6): 711-719. DOl: https:// doi.org/10.12820/rbafs.v.18n6p711. 